\title{
Restauradores Sin Fronteras: el patrimonio como motor de desarrollo sostenible
}

\author{
Asociación Restauradores Sin Fronteras (A-RSF) \\ Un patrimonio bien conservado es síntoma de resurgimiento \\ social y económico. \\ Javier Gámez Sánchez, presidente de A-RSF
}

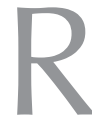

estauradores Sin Fronteras (A-RSF) es una organización no gubernamental [de cooperación cultural] para el desarrollo (ONGD), de carácter internacional, sin ánimo de lucro, abierta a cualquier persona o entidad, que nace en 1999, actúa de forma independiente a criterios políticos, religiosos o económicos y cuya sede central está en Madrid, España (Figura 1). Su misión fundamental consiste en realizar acciones que, al promover la utilización del patrimonio como motor para el desarrollo sostenible, beneficien social, económica y culturalmente a las comunidades y los países menos favorecidos. Así, la premisa al centro del trabajo de nuestra asociación es plantear que la puesta en valor y la gestión del legado cultural y natural de una población son generadoras de riqueza y de mejora en sus condiciones de vida, tanto al coadyuvar en el uso racional de sus recursos como al consolidar su identidad en un marco de respeto a la diversidad cultural.

Para A-RSF el patrimonio no está aislado del contexto socioeconómico y cultural, sino, por el contrario, forma parte, en diferentes medidas, de todas las actividades de una comunidad, por lo tanto para generar un desarrollo sostenible basado en la recuperación de nuestra herencia cultural y natural, lo que precisa actuar desde diferentes ámbitos: económico, social, educativo, político. Sólo de este modo el patrimonio se convierte en fuente y muestra de desarrollo.

\section{Objetivos específicos}

A-RSF, como se ha dicho, concentra sus esfuerzos en el uso del patrimonio cultural para promover el desarrollo sostenible de las comunidades menos favorecidas y tiene los siguientes objetivos específicos:

El desarrollo económico y social. El patrimonio cultural puede ser motor generador de ingresos —en algunos casos, la fuente principal-, al que hay que potenciar, para que sea un recurso sostenible, por medio de la implicación de la población beneficiaria en su gestión y mantenimiento. La rehabilitación del patrimonio, como el caso de los inmuebles, beneficia directamente a la población, que ve prosperidad en sus condiciones de vida, y aumenta, 


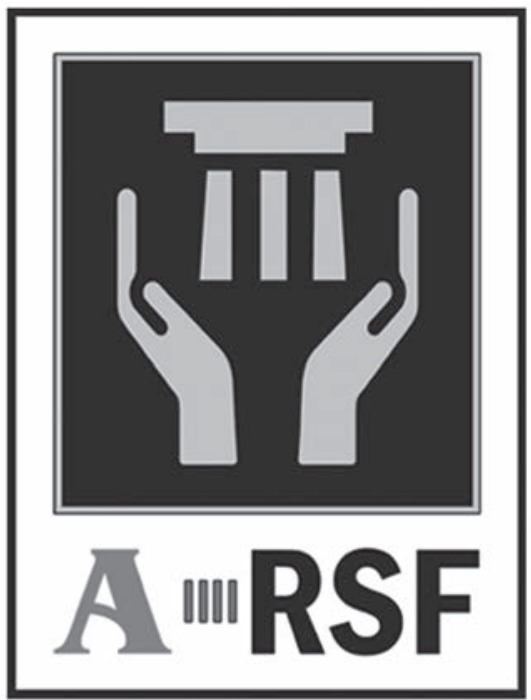

FIGURA 1. Logotipo de Restauradores Sin Fronteras (cortesía: A-RSF).

principalmente cuando se trata de centros históricos, la valoración de los conjuntos patrimoniales. La rehabilitación y la recuperación del patrimonio atraen inversiones públicas y privadas que alientan el desarrollo económico y social, mediante la generación de empleo y la mejora de las infraestructuras.

El aumento de las capacidades humanas e institucionales. La formación y capacitación en conservación y gestión del patrimonio cultural es una pieza esencial en los procesos de cambio y desarrollo locales, en tanto que proporciona conocimientos que luego podrán desarrollarse in situ y favorecer una mayor participación y un control comunitarios. El fortalecimiento institucional y el apoyo a la formación técnica, así como el asesoramiento y la consultoría para la realización de planes de manejo de centros históricos y ordenamientos territoriales, son algunos de los aspectos en los que se debe incidir para la sostenibilidad de los proyectos.

La protección de la identidad y diversidad cultural. La valoración del patrimonio cultural por parte de la población local es determinante para consolidar la identidad cultural como generadora de cohesión social, del aumento de la autoestima de la población y del sentido de pertenencia de las comunidades. La preservación de la identidad en un esquema de respeto a la diversidad cultural es un valor que hay que proteger ante la inminente pérdida, fruto de la globalización, de tradiciones locales.

La mejora de la gobernabilidad. El trabajo con las administraciones públicas encargadas de la protección del patrimonio cultural contribuye a aumentar y fortalecer los órganos de gestión local, así como a propiciar la de participación.

\section{Líneas de acción}

Para alcanzar estos objetivos, en los últimos años las acciones de A-RSF se han centrado en cuatro líneas principales:

Formación: proyectos dirigidos a la capacitación de personal local, que van desde la atención a la población más pobre o excluida hasta la formación de universitarios y técnicos en instituciones públicas

En este aspecto, A-RSF ha trabajado desde hace cinco años en el proyecto formativo de conservadores $y$ generación de movimientos sociales

restauradores de bienes culturales en Orán (Argelia), como estrategia para insertar laboralmente a los jóvenes, que conforman uno de los sectores más desamparados de la población argelina (Figura 2). Esta iniciativa busca, además, fomentar la promoción y la consolidación de técnicas de puesta en valor, y de desarrollo sostenible, en el campo de la conservación del patrimonio histórico y cultural de los pueblos, como herramienta activa para la mejora de la calidad de vida de la población local.

Sensibilización: acciones destinadas a promover e inculcar la protección del patrimonio cultural como un recurso para el desarrollo. Al igual que en la línea de trabajo referente a la formación, el abanico de beneficiarios va de la población local, haciendo hincapié en el trabajo con los niños, al fortalecimiento institucional de las autoridades competentes

Como ejemplo de esta línea destaca el proyecto "Guardianes del Patrimonio": programa de sensibilización social en Ciudad Velha, Cabo Verde, declarada Patrimonio de la Humanidad por la UNESCO en 2009, donde en los años previos a dicha declaración se realizó una fuerte inversión para rehabilitar los inmuebles más emblemáticos de la localidad, aun-

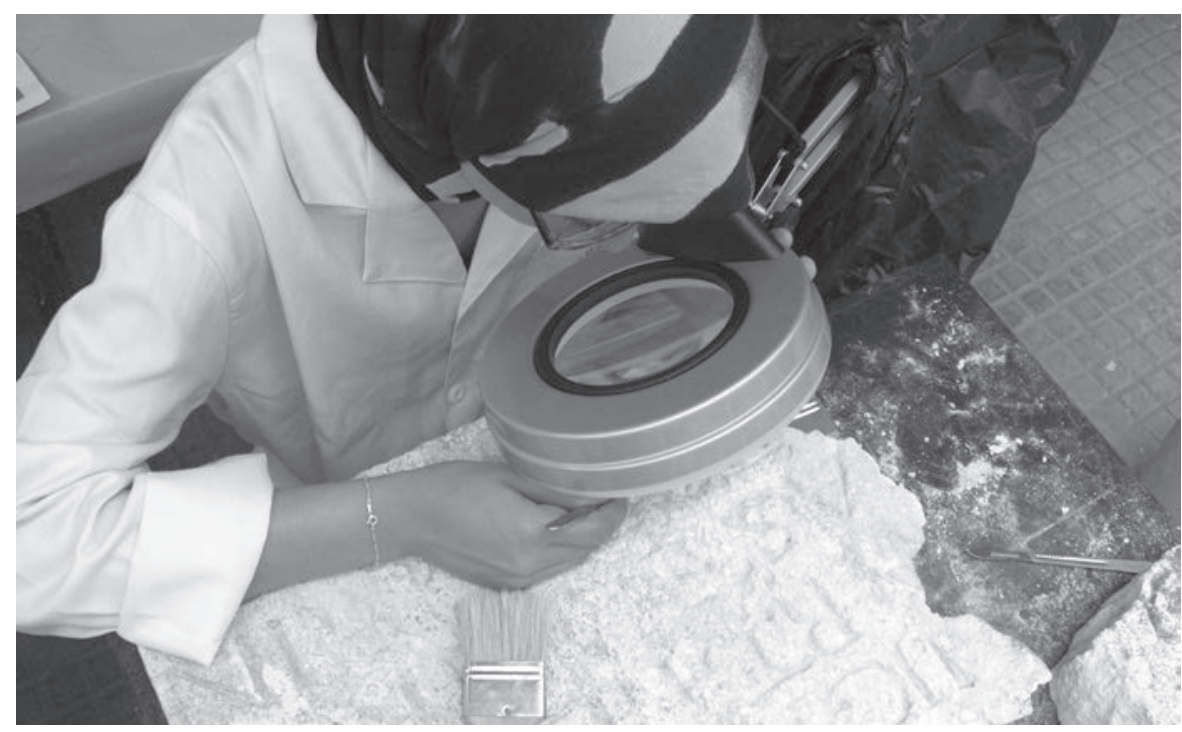

FIGURA 2. Alumna de la especialidad de Conservación y Restauración de Bienes Culturales, Orán, Argelia (Fotografía cortesía: A-RSF). 
que no se trabajó directamente con sus habitantes. En reparo de esta omisión, en 2009 A-RSF involucró a sectores comunitarios locales con el fin de fomentar la identificación y la valoración de su rico patrimonio cultural, para lo cual se diseñó un programa de sensibilización y capacitación que incorporó a los tres núcleos poblacionales de Ciudad Velha: niños, jóvenes y adultos, en la realización de diversas actividades y talleres colectivos.

Asistencias y estudios técnicos: en este aspecto las iniciativas se enfocan en realizar asesorías puntuales, estudios y planeamientos con metodología científica, para después pasar a su ejecución.

Sirva de ejemplo la asesoría realizada para la gestión del patrimonio de la ciudad de Orán, cuyos principales objetivos fueron acompañar el proceso de creación de la Unidad Técnica de Gestión Urbanística y capacitar a sus técnicos. Esto hizo posible tanto dar seguimiento al desarrollo y a la puesta en práctica de la figura de planeamiento urbano, así como proporcionar guía a particulares sobre aspectos patrimoniales en relación, por ejemplo, a procesos de rehabilitación de edificios residenciales. En esta iniciativa también se trabajó en el diseño de estrategias realistas que redunden en la eficacia de los servicios urbanos del barrio histórico de la ciudad.

Ejecución: proyectos globales de intervención en los que, por lo general, se cuenta con varios de los componentes y temáticas arriba mencionados.

El "Proyecto Gestión y Puesta en Valor del Patrimonio Cultural de Pomacocha, Perú", cuyo objetivo es mejorar las capacidades sociales, económicas y culturales de la comunidad local, se encuadra perfectamente en esta línea de acción. La elección de este núcleo poblacional surgió como iniciativa de lucha contra la pobreza en la zona, y planteó la necesidad de incorporar una vi- sión integral y participativa de la comunidad campesina de Pomacocha, caracterizada por su ubicación en agrícola, más deprimidas de la región andina.

La poca o escasa afluencia de visitantes nacionales y extranjeros a Pomacocha, sede de un importante legado cultural sudamericano, como la iglesia de Santa Clara del siglo XVII, ha generado su aislamiento de la economía regional, situación que movió a A-RSF a desarrollar un proyecto en la zona desde 2007. Su objetivo es generar actividades e iniciativas centradas en el desarrollo económico y social por medio de la valoración y la recuperación del patrimonio y la cultura locales, y apunta a lograr procesos de desarrollo, personal y colectivo, de autoestima. Asimismo, se ha impulsado tanto la capacitación, que genera un efecto de empoderamiento de la población local, como la creación de empleos temporales y permanentes relacionados con temas de valoración, conservación y uso sostenible del bien patrimonial. En la actualidad, esta iniciativa consiste de cinco componentes estratégicos que interactúan entre sí: educación y patrimonio local para elevar los niveles de cauna de las zonas, mayoritariamente

lidad en la formación de la comunidad; recuperación y conservación del patrimonio cultural, específicamente, de las portadas renacentistas de la iglesia de Santa Clara, y la rehabilitación del molino de época virreinal para su uso tradicional; capacitación y generación de riqueza, para reactivar y fortalecer la economía de la población; sostenibilidad y participación ciudadana, para propiciar la incorporación y apropiación del proyecto; y finalmente la elaboración, a través de un proceso de gestión participativa - consensuado entre los diferentes sectores-, para la operación de un circuito turístico cultural y ambiental que difunda la riqueza patrimonial local (Figura 3 ).

\section{Funcionamiento interno}

A-RSF ha establecido un mecanismo, en el que participan distintos sectores de la asociación, de toma de decisiones consensuadas y rápidas, con lo que se propicia que las resoluciones cuenten con el aval de la mayoría de la organización y se evita que sean unilaterales. Para asegurar una adecuada coordinación, existe una sede central en San Sebastián de los Reyes, Madrid (España), que trabaja en colaboración con los diferentes gru-

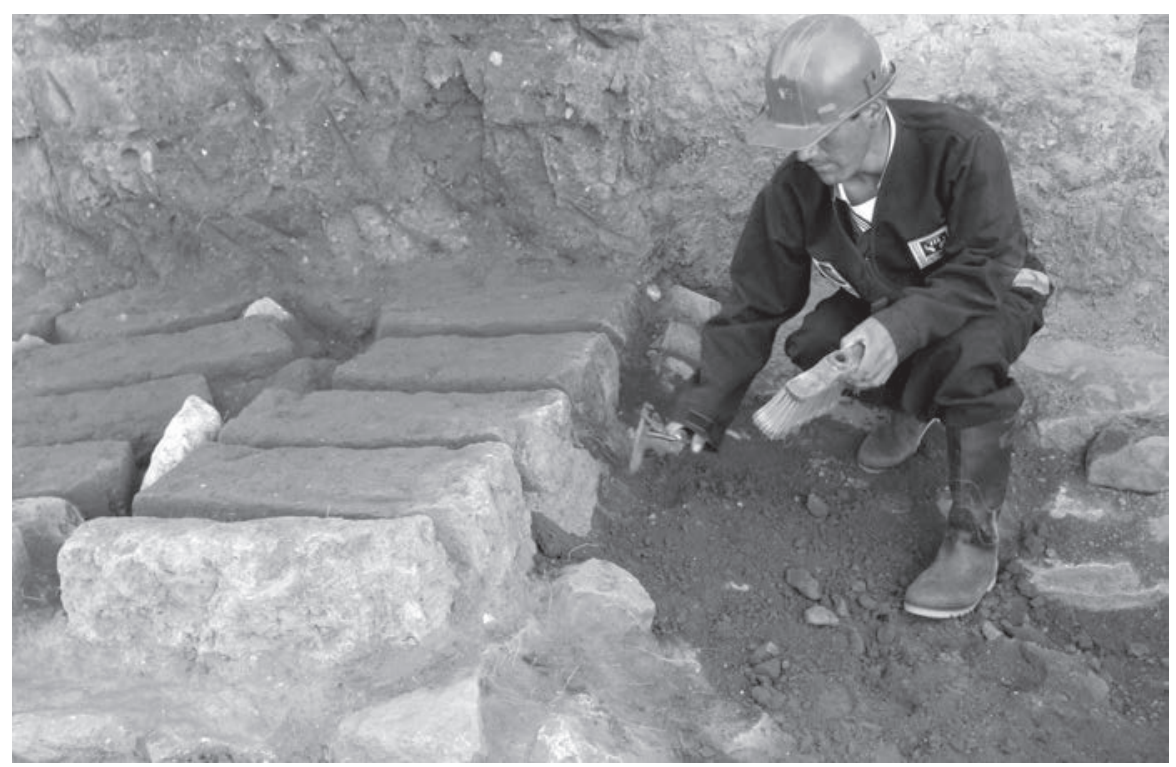

FIGURA 3. Un trabajador fabrica adobes para la rehabilitación de la iglesia de Santa Clara, Pomacocha, Perú (Fotografía cortesía: A-RSF). 


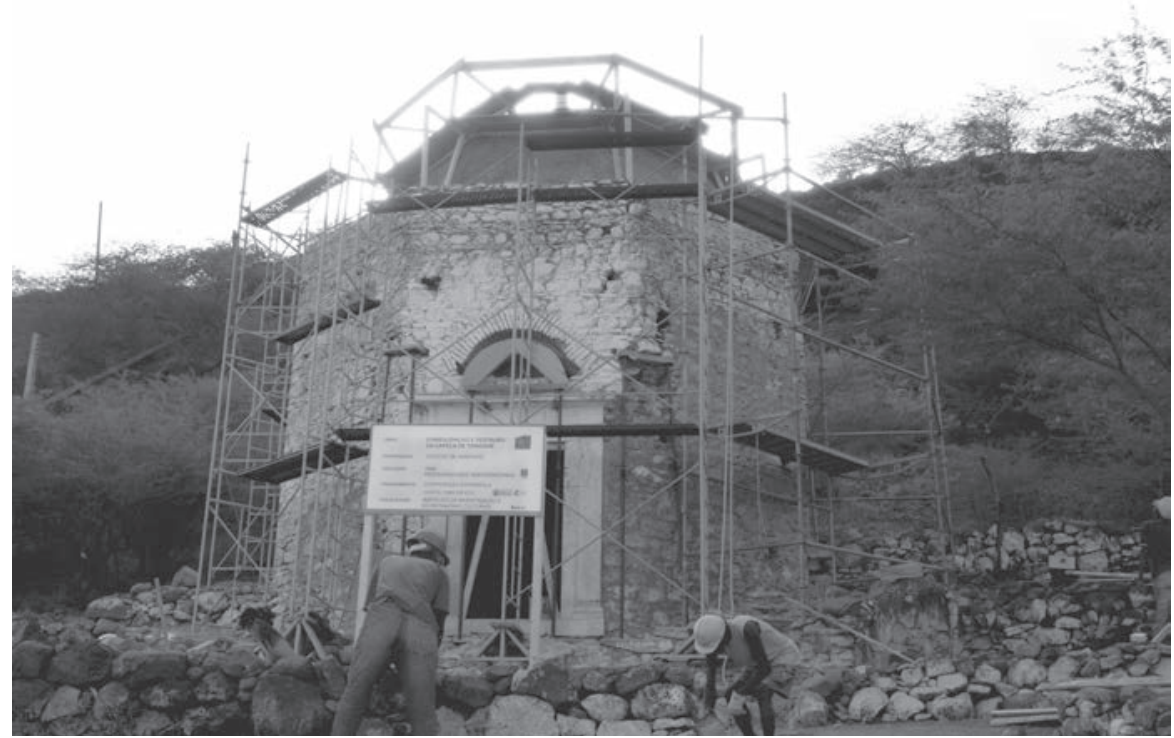

FIGURA 4. Proceso de rehabilitación de la capilla de Trindade, Isla de Santiago, Cabo Verde (Fotografía cortesía: A-RSF).

pos de Restauradores Sin Fronteras existentes - al día de hoy- en México, Perú, Colombia, Venezuela, Portugal y Costa Rica (Figura 4).

El máximo órgano de decisión en A-RSF es la Junta Directiva, que todos los socios eligen cada cuatro años en asamblea general. El Consejo Internacional de A-RSF, constituido por dos representantes de cada grupo, electo por la Junta Directiva de su país, se reúne en pleno al menos una vez al año, con el fin de intercambiar ideas y alcanzar una unidad de criterios para que, así, toda la organización siga una estrategia común.

\section{Financiamiento}

Desde su creación, Restauradores Sin Fronteras ha obtenido sus fondos principalmente por medio de tres vías:

Convocatorias anuales destinadas a proyectos de cooperación para el desarrollo de entidades como la Agencia Española de Cooperación Internacional al Desarrollo (AECID), comunidades autónomas, fundaciones, ayuntamientos, etcétera

Aportaciones de socios (particulares, museos, fundaciones, empresas)
Contribuciones privadas de empresas vinculadas directamente con la ejecución de algún proyecto concreto

Además, se busca mantener una buena política de transparencia, tanto interna como externa, la cual se garantiza mediante un cuidadoso trabajo, realizado internamente por el Área de Gestión y Administración de A-RSF con el apoyo de auditores externos, para tener una gestión y un control correctos de los recursos.

\section{Reflexiones y perspectivas}

En estos momentos de crisis mundial, la cooperación internacional para el desarrollo se ve gravemente afectada por importantes recortes económicos, situación que, naturalmente, también repercute en el curso de proyectos como los que ejecuta Restauradores Sin Fronteras, por lo que las expectativas de crecimiento, de estabilidad y de equilibrio no parecen muy esperanzadoras. Aunque es indudable que la articulación del patrimonio cultural como eje para el desarrollo constituye un motor clave para mejorar la calidad de vida de las poblaciones locales, ésta todavía no es una vía que las acciones de desarrollo gubernamental consideren como prioritaria. Pese a que la sociedad organizada comienza a asumir algunas responsabilidades, aún son pocas las organizaciones que, como A-RSF, trabajan con fundamento en esta perspectiva.

No obstante, lo anterior no debe ser motivo para flaquear en nuestros empeños. Debemos tomar en cuenta que uno de los principales ingresos económicos de varios países latinoamericanos, como Perú o México, es el turismo cultural. ARSF considera que las inversiones en este campo tendrían no sólo que ser mayores, sino también enmarcarse desde un planeamiento sostenible que, además de beneficiar a las comunidades, las incluya como actores activos en los procesos de toma de decisiones respecto de su patrimonio. En este sentido nuestra organización promueve una visión integrada de turismo y cultura, campos que no siempre van de la mano e incluso suelen gestionarse de manera independiente en diferentes ministerios, lo que evidencia la falta de una planificación conjunta. Creemos que acciones inconexas y con objetivos diferentes deben armonizarse $y$, así, colaborar conjuntamente para una cabal consecución de sus fines sociales.

Para A-RSF, los diferentes profesionales que trabajamos sobre el patrimonio tenemos que estar conscientes de que nuestros proyectos, si queremos garantizar la sostenibilidad del patrimonio y definir correctamente la planeación y las estrategias previas de cualquier intervención, deben incluir la participación social: de no lograrla - estamos convencidos de ello- no habrá apropiación y valoración del patrimonio cultural intervenido. Por tanto, las acciones de A-RSF buscan que en los próximos años nuestra profesión se encamine a que nuestros monumentos históricos sean físicamente salvados y que ello imprima el alma de las comunidades que los legarán a las nuevas generaciones. 


\section{Resumen}

La presente contribución describe la misión de Restauradores Sin Fronteras (A-RSF), organización no gubernamental para el desarrollo (ONGD), con el fin de detallar sus objetivos específicos y líneas de acción, todos ellos encaminados a promover la articulación del patrimonio como un motor para el desarrollo sostenible en comunidades menos favorecidas. Se mencionan algunos de los proyectos realizados en 2011 y se reflexiona sobre algunas perspectivas a futuro, particularmente en el ámbito latinoamericano. Para mayor información, consúltese la página web de la asociación: [www.a-rsf.org].

\section{Palabras clave}

A-RSF, cooperación para el desarrollo, patrimonio cultural, ONGD.

\section{Abstract}

This contribution describes the structure and mission of Restauradores Sin Fronteras (A-RSF) (Restorers Without Borders), a non-governmental development organization (NGDO), detailing its specific objectives and lines of action, all aimed at promoting cultural heritage as a driving force for sustainable development in disadvantaged communities. Some of the projects implemented in 2011 are mentioned and we also reflect upon some future prospects, especially in Latin America. For more information, visit the association's website at [www.a-rsf.org].

\section{Keywords}

A-RSF, development cooperation, cultural heritage, NGDO.

Título en inglés: "Restorers Without Borders: cultural heritage as a driving force

for sustainable development" 